\title{
FOAM-ON-TILE IMPACT MODELING FOR THE STS-107 INVESTIGATION
}

\author{
R. F. Stellingwerf*, J. H. Robinson** \\ S. Richardson***, S. W. Evans***, R. Stallworth***, M. Hovater*** \\ *Stellingwerf Consulting, 2229 Loma Linda, Los Alamos, NM 87544 \\ **Morgan Research Corp., 4811-A Bradford Dr., Huntsville, AL 35806 \\ ***NASA Marshall Space Flight Center, Huntsville, AL 35812
}

\begin{abstract}
$\underline{\text { ABSTRACT }}$
Following the breakup of the Space Shuttle Columbia during reentry a NASA/Contractor investigation team was formed to examine the probable damage inflicted on Orbiter Thermal Protection System elements by impact of External Tank insulating foam projectiles. Our team was to apply rigorous, physics-based analysis techniques to help determine parameters of interest for an experimental test program, utilize validated codes to investigate the full range of impact scenarios, and use analysisderived models to predict aero-thermal-structural responses to entry conditions. We were to operate on a non-interference basis with the Impact Testing Team, and were to supply significant findings to that team and to the Orbiter Vehicle Engineering Working Group, being responsive to any solicitations for support from these entities.
\end{abstract}

The authors formed a working sub-group within the larger team to apply the Smooth Particle Hydrodynamics code SPHC to the damage estimation problem. Numerical models of the Orbiter's tiles and of the Tank's foam were constructed and used as inputs into the code. Material properties needed to properly model the tiles and foam were obtained from other working sub-groups who performed tests on these items for this purpose. Two- and three-dimensional models of the tiles were constructed, including the glass outer layer, the main body of LI-900 insulation, the densified lower layer of LI-900, the Nomex felt mounting layer, and the Aluminum 2024 vehicle skin. A model for the BX-250 foam including porous compression, elastic rebound, and surface erosion was developed. Code results for the tile damage and foam behavior were extensively validated through comparison with Southwest Research Institute foam-on-tile impact experiments carried out in 1999. These tests involved small projectiles striking individual tiles and small tile arrays.

Following code and model validation we simulated impacts of larger foam projectiles on the examples of tile systems used on the Orbiter. Results for impacts on the main landing gear door are presented in this paper, including effects of impacts at several angles, and of rapidly rotating projectiles. General results suggest that foam impacts on tiles at about $500 \mathrm{mph}$ could cause appreciable damage if the impact angle is greater than about 20 degrees. Some variations of the foam properties, such as increased brittleness or increased density could increase damage in some cases. Rotation up to $17 \mathrm{rps}$ failed to increase the damage for the two cases considered. This does not rule out other cases in which the rotational energy might lead to an increase in tile damage, but suggests that in most cases rotation will not be an important factor.

\section{BACKGROUND}

Project Objectives

The External Tank (ET) Program is interested in characterizing permissible debris generation limits for the Spray On Foam Insulation (SOFI) used on the ET. To aid in this determination, a better understanding is needed of what roles density and size of released fragments of SOFI, and corresponding impact energies, have on various materials, including the Space Shuttle Orbiter ceramic tiles. Smooth Particle Hydrodynamics (SPH) is a fully Lagrangian, fully conservative computational technique for

Copyright $\mathbb{2 0 0 4}$ by the American Institute of Aeronautics and Astronautics, Inc. No copyright is asserted in the United States under Title 17, U.S. Code. The U.S. Government has a royalty-free license to exercise all rights under the copyright claimed herein for Governmental purposes. All other rights are reserved by the copyright owner. 
hydrodynamic problems that may be able to characterize the damage induced by an ET foam projectile during impacts at high velocities. Details of SPH are given in references 1-7.

Previous analyses employing the Smooth Particle Hydrodynamics Code (SPHC) characterized hypervelocity ( $>2 \mathrm{~km} / \mathrm{s}$ ) impact damage to tethers ${ }^{8,9}$ and to a thermal protection system of Alumina Enhanced Thermal Barrier $(\mathrm{AETB})^{10}$. The impact velocity in the present case was in the "high velocity" category of several hundred $\mathrm{m} / \mathrm{s}$. SPH analysis has been done previously for materials in this velocity regime ${ }^{11}$.

An extensive series of experiments of foam impacting Space Shuttle tiles was published by Goodlin $^{12}$ at Southwest Research Institute (SwRI). We used these experiments to validate the SPHC models of foam-on-tile impacts and to determine the accuracy and limitations of this modeling approach.

\section{Thermal Tiles}

Figure 1 shows a published photograph of a damaged thermal tile from the Space Shuttle Columbia. The tile is probably from the undersurface of a wing, and consists of an outer coating of reaction cured glass (RCG) (black in the photo), and an inner core of LI-900 (a Lockheed-Martin product consisting of "rigidized fiberous silica" with a density of 9 $\mathrm{lb} / \mathrm{ft}^{3}$ ) (white). The damage seen here is typical of extreme heating and/or impact damage that melts or shatters the tile's coating, exposing the low density inner material to "gouging" from a low angle collision with high speed debris. The RCG coating can withstand temperatures in excess of 2000 degrees F. Although the tiles vary widely in shape and size, this one appears to be the most common variety, which is 6 inches square and about 2 inches thick.

This tile almost certainly sustained damage during the accident, but it is known that tiles can be damaged during liftoff due to impacts with dislodged fragments of foam insulation from the External Tank. Such a collision was observed at about $81 \mathrm{~s}$ after liftoff of Columbia. The foam insulation on the External Tank is extremely porous light-weight material, but it can still cause damage to the fragile RCG coating if impacting with high kinetic energy. In fact, the damage predicted to occur from impacts with foam material resembles that seen in Figure 1.

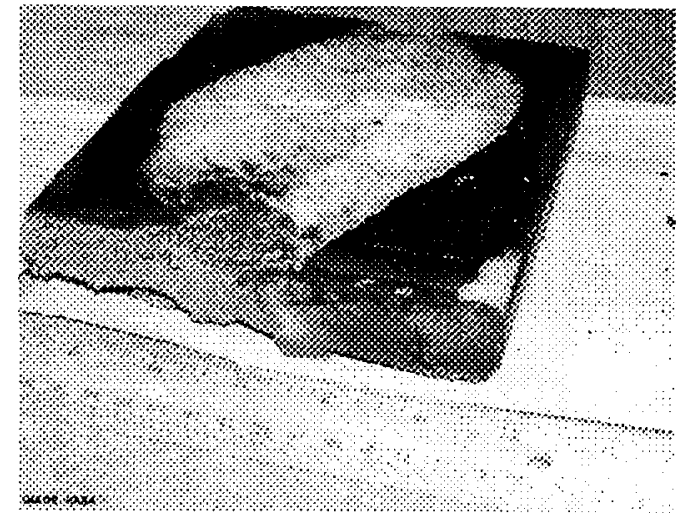

Figure 1. Thermal Protection Tile from the Space Shuttle Columbia recovered near Powell, Texas.

Figure 2 shows details of the tile TPS ${ }^{13}$. The "HRSI" (Heat Resistant Silica Insulation) is LI900 in this case, the RCG coating, Strain Isolation Pad (SIP), RTV adhesive, and an underlying "skin" or wing segment are shown in the illustration. The thickness of the SIP is not $0.4 \mathrm{~mm}$ as indicated, but $0.4 \mathrm{~cm}$ in most cases.

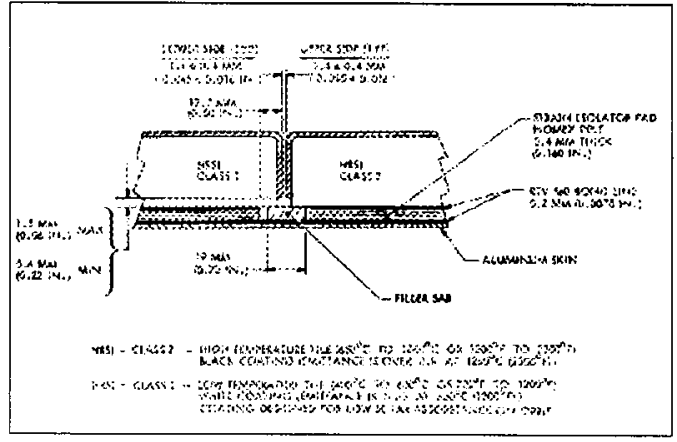

Figure 2. Schematic of the LI-900 tile configuration.

The "Filler Bar" is a section of Nomex felt that seals the gap between the tiles. The SIP pads and the gaps allow flexure of the wing without dislodging tiles due to pinching or twisting.

\section{SPHC Tile Models}

The main challenge to designing a model of the LI-900 tile is the treatment of the thin layers of RCG (glass coating) and RTV (adhesive). Tests showed that the RCG layer could be modeled by a thin layer of small SPH particles with the right RCG properties. Usually, only a single layer could be modeled. This is sufficient to provide the effects of the RCG mass density and surface texture, but has the drawback of not modeling the internal details of the RCG correctly. For the present set of models, the RTV is not modeled 
explicitly, rather its bond strength is treated by the code as an edge effect between regions. Although the damage did not extend to the RTV layer in the tile for any of the current models, the treatment of the edge effects in the code is a conservative one, which could be relaxed if necessary if the damage had reached the RTV boundaries.

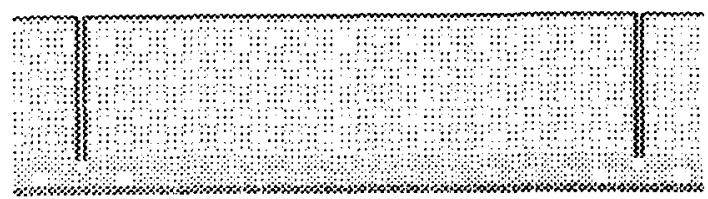

Figure 3. SPHC LI-900 tile configuration.

Figure 3 shows the SPHC implementation of the tile structure. The upper layer is the RCG, and the lowest layer is the $\mathrm{Al} 2024$ "skin," which represents the wing surface. Above the aluminum are layers of SIP and gap fillers, and above these the densified layer of the LI- 900 . The thickness of this tile is 1.8 inches.

The tiles used in the SwRI experiments vary slightly from the above figure. The thickness used was 2 inches, a SIP layer was not used, and the base was a 0.25 inch thick steel plate. The

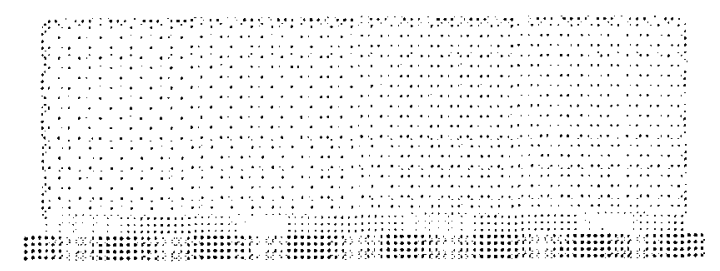

Figure 4. SPHC SwRI LI-900 tile.

corresponding SPHC setup is shown in Figure 4, which was the configuration used for the validation model.

\section{SWRI EXPERIMENTS}

Goodlin ${ }^{12}$ presents results of an extensive series of foam-on-tile impact experiments at Southwest Research Institute. Five different shapes of foam projectiles were used, as shown in Figure 5. The projectile dimensions in inches were: blocks of $1 \times 1 \times 3 ; 1 \times 1 \times 1 ; 0.89 \times 0.89 \times 0.25$; cylinders of $3 / 8$ diameter, and either 1 or 3 long. Weights of each of the projectiles were given, from which we inferred foam densities of 0.034 to $0.039 \mathrm{~g} / \mathrm{cm}^{3}$. Since all of the projectiles were composed of NCFI 24-124 foam, they should have had the same density, assumed to be close to that of BX250 foam, which is $0.038 \mathrm{~g} / \mathrm{cm}^{3}\left(2.4 \mathrm{lb} / \mathrm{ft}^{3}\right)$. This is the only foam property given in the report. The $15 \%$ variation in the computed densities is unexplained, but is probably due to inaccuracy in the measurements, though no measurement accuracy is specified in the report. Each shot reports a foam weight, but the weights for a given shape are all identical, so it is unlikely that individual projectiles were actually weighed at the time of the shot. The SPHC validation models assume a density of $2.4 \mathrm{lb} / \mathrm{ft}^{3}$ and dimensions exactly as given for all shots.

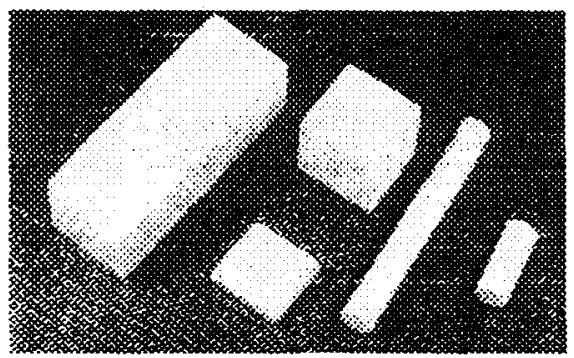

Figure 5. Foam projectiles used in the SwRI experiments.

The experiments used actual LI-900 flight tiles as well as a few fresh "engineering" tiles as targets, covered the velocity range of 400-1800 $\mathrm{ft} / \mathrm{s}$, and had impact angles of 10-60 degrees from the horizontal. The results were measured size and depth of the tile damage crater, photos of the impact and of the damaged tile, and details of each shot.

Since no substantial analyses of the results were presented in the SwRI report, we summarize the results here. Of the approximately 200 shots reported, we find that 65 are useful for data analysis. The others either had zero damage (only the fastest of each zero damage series determined the damage threshold), had the projectile break up in flight, or showed some other anomaly. Plotting crater depth versus velocity should show a clear trend, and constitutes the main result of this survey. Figure 6 shows such a plot for all shots, scaled on mass $^{1 / 3}$ and $(L D)^{1 / 3}$. A fitting line is shown for reference, and illustrates the main features of the data: i) a damage threshold in velocity, and ii) a power law type increase of depth with velocity 


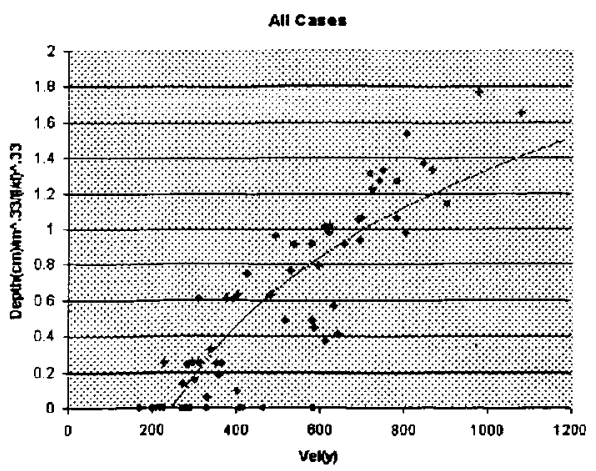

Figure 6. Crater depth versus normal velocity for all clean shots.

(at higher velocities this is a $2 / 3$ power ${ }^{14}$ ). The data also show an "inherent" but unexplained scatter of about $+/-0.5 \mathrm{~cm}$ in crater depth. This scatter is mainly due to fracture of the foam during acceleration and irregular fracture of the RCG at initial impact. Variations in the wear and existing damage to the tiles may also contribute. In some cases tiles were shot multiple times, which may also affect the results.

\section{MODEL RESULTS}

The model runs consisted of the LI- 900 tile model described above impacted by BX-250 foam, which has the same density as the NCFI 24-124 foam used in the experiments, and which is believed to have similar material properties. All material properties are for "standard temperature and pressure" conditions. This is appropriate for the experiment, but may not apply to actual Orbiter events.

Shot 52B was chosen as an initial test case. This is a shot with good data and no visible breakup of the projectile during launch. The projectile was $1 \times 1 \times 3$ inches, with the long dimension along the direction of travel. The impact angle was 23 degrees from horizontal, and the velocity was $1588 \mathrm{ft} / \mathrm{s}$. The measured crater depth for this shot was 0.8 inches $(2.03 \mathrm{~cm})$, and the damaged area on the surface of the tile measured $1.1 \times 4$ inches (see Figure 7). The camera angle used in the experiment seems to have been selected to measure the impact velocity and little else, so we have only one tantalizing glimpse of the impact, as shown in Figure 8, corresponding to about $0.1 \mathrm{~ms}$ into the event.

\section{3-D Test Case}

The four frames of Figure 9 show the 3-D model of shot $52 \mathrm{~B}$ in progress, including details

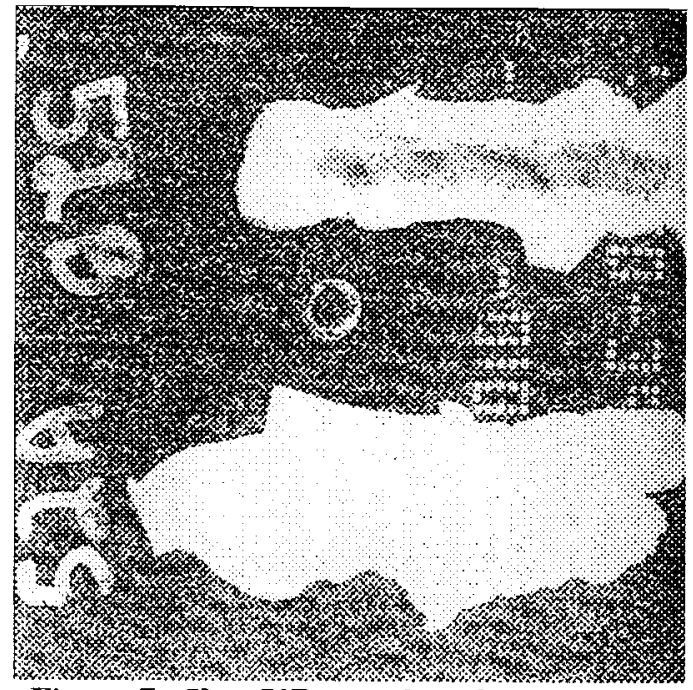

Figure 7. Shot 52B - surface damage photo, top impact point.

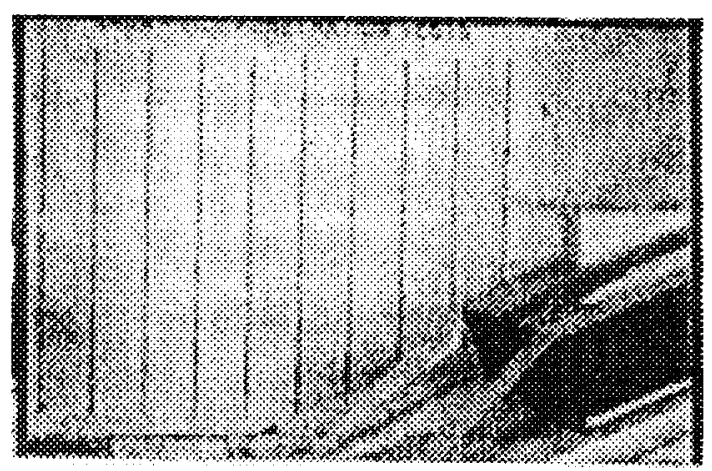

Figure 8. Shot 52B, one frame of 8 shown in the SwRI report, but the only one showing the impact in progress.

of the impact. The foam debris is "dust" in this shot and will expand into a dispersed cloud at later times. At $80 \mathrm{~ms}$ the damaged area was about 1.5 inches wide and 3.6 inches long.
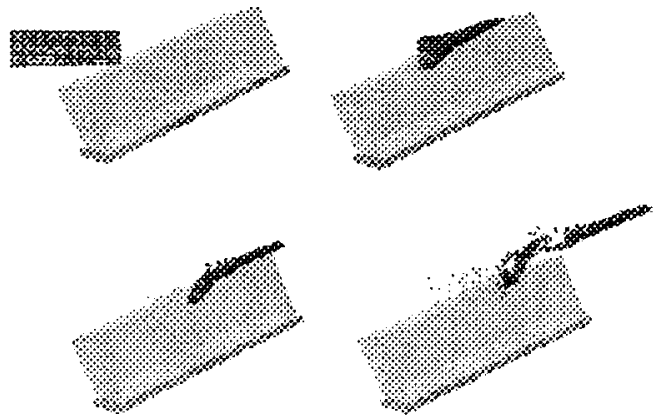

Figure 9. Shot 52B, 3D model, experimental view angle, cut-away view, at $0 \mathrm{~ms}, 0.20 \mathrm{~ms}$, $0.30 \mathrm{~ms}$ and $0.50 \mathrm{~ms}$. 


\section{2-D vs. 3-D Comparison}

Since the foam has minimal lateral spreading during the impact, and a Poisson ratio near zero, the 2-dimensional model was expected to be a good approximation to the 3-D experiment. The exception would be the lateral dissipation in the tile, and thus the 2-D analysis may overestimate the damage to some extent. On the other hand, the 2-D model would have better resolution than the 3-D model, and should show more details of the impact. To evaluate the accuracy of a 2-D model, we compare a two-dimensional slice of the 3-D model, Figure 10, with the 2-D model result, Figure 11. The overall results compare

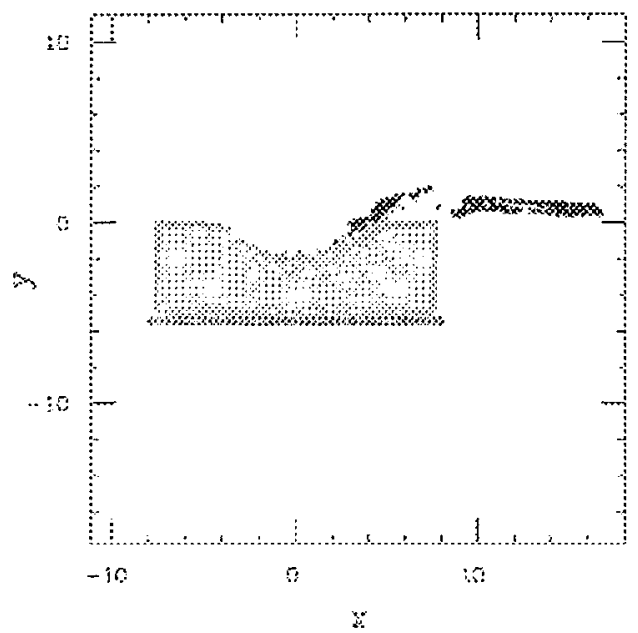

Figure 10. Shot 52B, slice through 3D model, at $0.50 \mathrm{~ms}$.

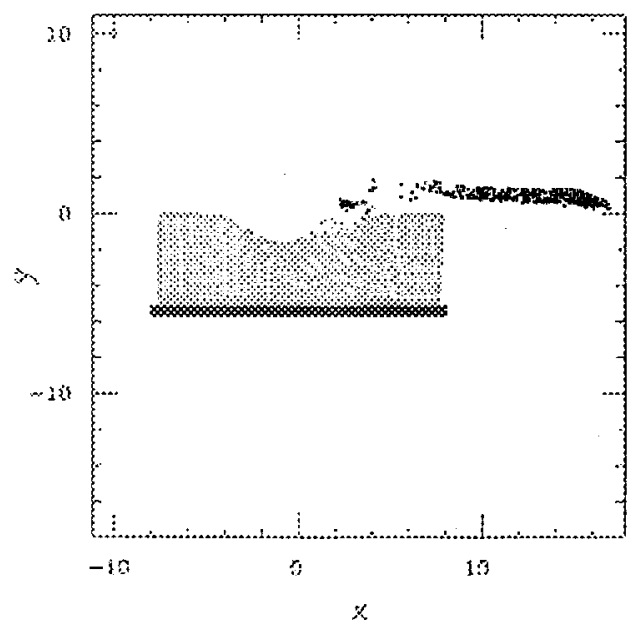

Figure 11. Shot 52B, 2D model, at $0.50 \mathrm{~ms}$.

closely, with crater depths nearly identical. The 2-D crater is somewhat Ionger than the 3-D result, and the debris cloud is slightly different. We concluded that 2-D models should yield accurate crater depths that can be compared to the experimental values. The accuracy of the 2-D result is higher for larger projectiles, and less for smaller projectiles.

\section{Effect of Stiff and Brittle Foam}

We ran a test to see what effects cryotemperatures might have on the foam, making following changes to the usual foam model: i) the max-elongation was reduced to 0.05 (brittle); ii) the yield stress, tensile stress, and maximum tensile volumetric pressure were all increased by a factor of 50 (stiff). This is a larger change than we expect at ET temperatures. The result is shown in Figure 12. The changes in the foam properties have a large effect on the foam debris cloud. The crater in this case has a slightly different shape than seen in Figure 11, but the crater depth is only slightly deeper $(1.9 \mathrm{~cm}$, compared to $1.8 \mathrm{~cm}$ ). The damage seems to be more localized than before. The debris cloud is more dispersed at this time (the normal case disperses later in time), and contains several unfractured chunks of foam, probably due to the increased strength parameters.

We conclude that cryotemperatures will have a major effect on the foam debris cloud, but minimal if any effect on the crater depth and overall damage to the tile.

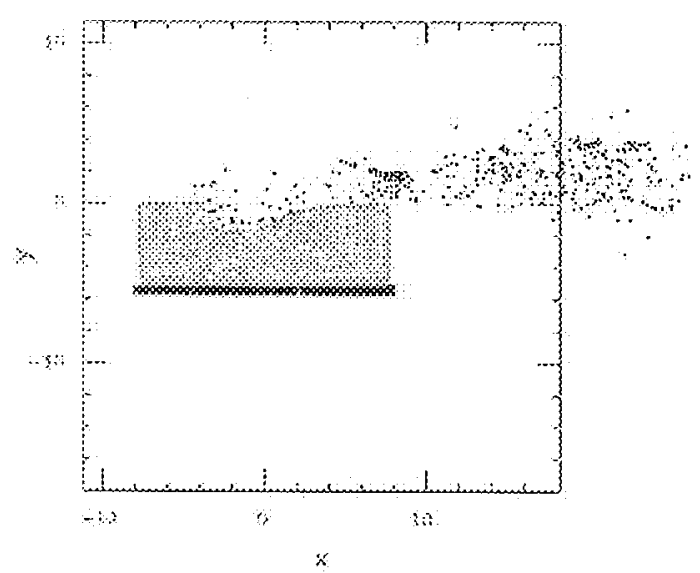

Figure 12. Shot 52B, 2-D cryotemperature test, at $0.50 \mathrm{~ms}$.

Tests at lower angles of incidence and velocities near $200 \mathrm{~m} / \mathrm{s}$ had the following results:

1. Reduction of the max-elongation to 0.042 without a change in strength produced a foam debris cloud composed nearly entirely of fractured dust. 
2. Reduction of the max-elongation to 0.042 with a factor of 2 increase in strength produced several large foam debris fragments.

3. Reduction of the max-elongation to 0.042 with a factor of 10 increase in strength produced a single foam fragment.

These conclusions are sensitive to the fracture model in the foam, which is complex and difficult to model. Therefore, these characterizations should be taken as general indications of what to expect, not as final results.

\section{MSFC Test Cases}

Three cases were proposed by NASA-Marshall Space Flight Center as test cases for the foamon-tile impact models. These are SwRI shots 24F, 55 and 104; their results are discussed below.

Shot $24 \mathrm{~F}$ was a $1 \mathrm{x} 1 \mathrm{x} 1$ inch projectile shot against a pristine tile at $1788 \mathrm{ft} / \mathrm{s}$ and 10 degrees from horizontal. The test shot damage had a depth of 0.1 inch $(0.254 \mathrm{~cm})$, and a horizontal extent of $2 \times 2.5$ inches. The 2 -D model run had a damage depth of $0.20 \mathrm{~cm}$, similar to the experiment. The 3-D model resulted in damage of almost identical depth, measuring 1.6x2.4 inches laterally. The actual experiment is slightly wider due to the flaking of the RCG layer, which is not well modeled by the single layer of particles in this simulation.

Shot 55 was a $1 \times 1 \times 3$ inch projectile shot at 1161 $\mathrm{ft} / \mathrm{s}$ and 30 degrees from horizontal. The test shot damage depth was 0.75 inch $(1.905 \mathrm{~cm})$. The 2D model run is shown in Figure 13, with damage depth of $1.8 \mathrm{~cm}$, again similar to the experiment. Substantial damage, including a diagonal shear fracture, is visible in the tile at later times.

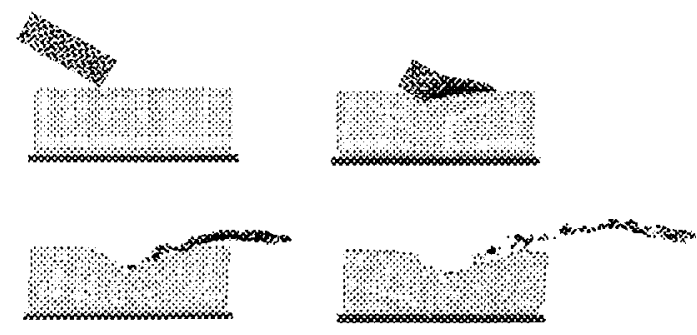

Figure 13. Model results for shot 55 at times $0,0.20,0.50$ and $0.80 \mathrm{~ms}$.
Shot 104 was a $0.375 \times 3$ inch cylindrical projectile shot at $1250 \mathrm{ft} / \mathrm{s}$ and 60 degrees from horizontal. The experimental damage depth was 0.9 inch $(2.29 \mathrm{~cm})$. The 2-D model run obtained a damage depth of $2.3 \mathrm{~cm}$, again nearly identical to the experiment.

All three test cases show agreement with the experimental crater depths to within $1 \mathrm{~mm}$.

\section{Test Series Results}

Ten cases were selected for detailed comparison with experimental results. These cases, along with the shot parameters, experimental crater depths, and model depths, are given in the following table. To help appreciate the accuracy of these results, the crater depth as computed by the "R\&C" formula derived by Richardson and $\mathrm{Chou}^{14}$ is given. It is based on an earlier set of foam-on-tile experiments shot at Texas $A \& M^{15}$. This is the formula believed to have been used to evaluate Columbia tile damage in January, 2003.

Table 1. SwRI Shuttle Tile Test Shots

\begin{tabular}{|c|c|c|c|c|c|c|c|}
\hline \multirow[t]{2}{*}{ Shot } & \multirow{2}{*}{$\begin{array}{c}\text { Proj } \\
\text { in }\end{array}$} & \multirow{2}{*}{$\begin{array}{l}\text { Vel } \\
\mathrm{ft} / \mathrm{s}\end{array}$} & \multirow{2}{*}{$\begin{array}{l}\text { Ang } \\
\text { deg }\end{array}$} & \multicolumn{2}{|c|}{ Depth } & \multirow{2}{*}{$\begin{array}{c}\text { SPHC } \\
\mathrm{cm}\end{array}$} & \multirow{2}{*}{$\begin{array}{c}\mathrm{R} \& \mathrm{C} \\
\mathrm{cm}\end{array}$} \\
\hline & & & & in & $\mathrm{cm}$ & & \\
\hline $24 \mathrm{~F}$ & $1 \times 1 \times 1$ & 1788 & 10 & 0.1 & 0.254 & 0.20 & -0 \\
\hline 49 & $1 \times 1 \times 3$ & 440 & 23 & 0.0 & 0.0 & {$[0.4]$} & -0 \\
\hline $50 \mathrm{~A}$ & $1 \times 1 \times 3$ & 723 & 23 & 0.2 & 0.51 & 0.7 & 1.08 \\
\hline $51 \mathrm{~B}$ & $1 \times 1 \times 3$ & 1356 & 23 & 0.63 & 1.60 & 1.5 & 3.02 \\
\hline $52 B$ & $1 \times 1 \times 3$ & 1588 & 23 & 0.80 & 2.03 & 1.8 & 3.57 \\
\hline 54 & $1 \times 1 \times 3$ & 803 & 30 & 0.08 & 0.20 & 1.3 & 2.13 \\
\hline 55 & $1 \times 1 \times 3$ & 1161 & 30 & 0.75 & 1.91 & 1.8 & 3.33 \\
\hline $59 \mathrm{~A}$ & $1 \times 1 \times 3$ & 1126 & 40 & 1.00 & 2.54 & 2.3 & 4.16 \\
\hline 103 & $3 / 8 \times 3$ & 1071 & 30 & 0.50 & 1.27 & 1.3 & 1.78 \\
\hline 104 & $3 / 8 \times 3$ & 1250 & 60 & 0.90 & 2.29 & 2.3 & 3.46 \\
\hline
\end{tabular}

Note 1: Shot 49 had a "no damage" result. This means the RCG coating on the tile was not cracked. The model showed an indentation of about $0.4 \mathrm{~cm}$, but an intact and unfractured RCG layer. The actual RCG may be able to recover from this type of perturbation.

Note 2: Shot 54 is the only case in which the code results do not agree with the experiment results - an error of $1.1 \mathrm{~cm}$, or $22 \%$ of the tile thickness. There is nothing abnormal noted concerning this shot, but careful examination of the results do suggest that the experimental result is low for some reason. For example, shot 93 , at 23 degrees and $870 \mathrm{ft} / \mathrm{s}$ would be expected to have smaller damage due to the shallower angle, but has a depth of 0.27 inch $(0.69 \mathrm{~cm})$. Shot 58 at 40 degrees and $801 \mathrm{ft} / \mathrm{s}$ is comparable to 54 , but has damage of 0.4 inch $(1.02 \mathrm{~cm})$. Both of these cases are consistent with the code result for shot 54, but not with the shallow experimental crater depth.

Excluding shot 54, the average deviation of the model vs. experiment results is $0.11 \mathrm{~cm}$, which is only $2 \%$ of the tile thickness. The average relative error (difference/depth) is $10.7 \%$. For comparison, the average error of the $\mathrm{R} \& \mathrm{C}$ 
formula is $0.95 \mathrm{~cm}$, which is about $20 \%$ of the tile thickness, and the average relative error for $\mathrm{R} \& \mathrm{C}$ is $67 \%$.

A comparison of these results can be seen in Figure 14, where the test data, the model results, and the $\mathrm{R} \& \mathrm{C}$ results are plotted. The model results are well within the scatter of the data set, whereas the $\mathrm{R} \& \mathrm{C}$ results fall generally far above the experimental depths.

\section{Engineering Tiles}

One feature of the SwRI study was the inclusion of "engineering" tiles, i.e. new, unused tiles, as well as a collection of shuttle tiles that had been

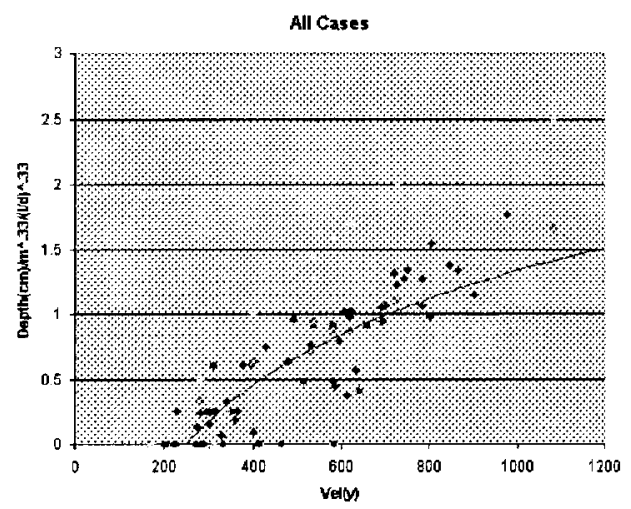

Figure 14. Plot of all cases showing experimental crater depths (diamonds), model results (squares), and " $\mathbf{R} \mathbf{C}$ " results (triangles).

used, and, in most cases, removed because of wear and tear. Such wear and tear could affect the strength of the tiles, and so the damage inflicted by a foam projectile. This subject was not addressed in the SwRI report, however, so we address it here.

Close inspection of the 3 inch foam projectile shots revealed that only three of them used engineering tiles and also produced acceptable results: $50 \mathrm{~A}, 101 \mathrm{~A}$, and 101B. These three shots were modeled, with results given in Table 2 .

It is interesting to note that the SPHC crater depths for these models are about $20 \%$ too deep. This may be an indication of the effect of using new tiles, since the model results do match the used tile sequences quite well. Caution is advised, however, in drawing such a conclusion from just three points, especially since all of these cases seem to have some sort of problem in the data set. Shot 50A had many cracks in the
Table 2. SwRI Engineering Tile Shots

\begin{tabular}{|c|c|c|c|c|c|c|c|}
\hline \multirow[t]{2}{*}{ Shot } & \multirow{2}{*}{$\begin{array}{c}\text { Proj } \\
\text { in }\end{array}$} & \multirow{2}{*}{$\begin{array}{l}\text { Vel } \\
\mathrm{ft} / \mathrm{s}\end{array}$} & \multirow{2}{*}{$\begin{array}{l}\text { Ang } \\
\text { deg }\end{array}$} & \multicolumn{2}{|c|}{ Depth } & \multirow{2}{*}{$\begin{array}{c}\text { SPHC } \\
\mathrm{cm}\end{array}$} & \multirow{2}{*}{$\begin{array}{c}\mathrm{R} \& \mathrm{C} \\
\mathrm{cm}\end{array}$} \\
\hline & & & & in & $\mathrm{cm}$ & & \\
\hline $50 \mathrm{~A}$ & $1 \times 1 \times 3$ & 723 & 23 & 0.20 & 0.508 & 0.60 & 1.08 \\
\hline $101 \mathrm{~A}$ & $3 / 8 \times 3$ & 1400 & 30 & 0.58 & 1.47 & 1.80 & 2.35 \\
\hline $101 \mathrm{~B}$ & $3 / 8 \times 3$ & 1317 & 30 & 0.50 & 1.27 & 1.50 & 2.21 \\
\hline
\end{tabular}

pre-shot tile. Shot 101A does not show a velocity picture sequence, and is not included in the final shot list (the velocity is probably suspect). Shot $101 \mathrm{~B}$ shows a small rotation after launch that could reduce the crater depth. We conclude that there is a slight indication of about a $20 \%$ degradation of the tile strength (as measured by crater depth) in the used tiles, as compared to new tiles, and that the models tend to fall closer to the used tile case for some reason. Probably, the RCG on the fresh engineering tiles was somewhat stronger than that of the used shuttle tiles, and stronger than the material parameters included in the models.

Fitting Formula for Crater Depth

We derived an improved version of the $R \& C$ formula that is more accurate for the SwRI results than the original, and matches the SPHC model results as well. Most of the scaling laws used in $\mathrm{R} \& \mathrm{C}$ have been retained. These could be checked by making many more model runs, but we have not performed these at this time. Our expression would probably be a better choice for making a quick estimate of tile damage, at least within the range of the experimental data. The new equation is:

$$
\begin{aligned}
& P=\frac{(0.0086)(L / d)^{0.67} d \rho_{p}{ }^{0.27}(V-\hat{V})^{0.67}}{S_{t}^{0.25} \rho_{t}{ }^{0.17}} \\
& \text { where } \\
& \qquad \begin{array}{l}
\mathrm{P}=\text { penetration depth (in) } \\
\mathrm{L}=\text { length of projectile (in) } \\
\mathrm{d}=\text { diameter or width of projectile (in) } \\
\left.\rho_{\mathrm{p}}=\text { density of projectile (lb/in }{ }^{3}\right) \\
V=\text { normal velocity (in/s) } \\
\hat{V}=\text { threshold normal velocity (in/s) : } \\
\quad \hat{V}=21.4 \sqrt{S_{t} / \rho_{p} L} \\
S_{\mathrm{t}}=\text { tile compressive strength (psi) } \\
\rho_{\mathrm{t}}=\text { tile density }\left(\mathrm{lb} / \mathrm{in}^{3}\right)
\end{array}
\end{aligned}
$$

The exponent and multiplier of $(\mathrm{L} / \mathrm{d})$ have been changed from 0.45 to 0.67 , and from 0.0195 to 
0.0086 , respectively, compared with the original $\mathrm{R} \& \mathrm{C}$ formula.

The accuracy of the new expression is illustrated in Figure 15, which is identical to Figure 14, except that the predictions made using our formula are shown as green o's. These values are as good as the model data for this purpose.

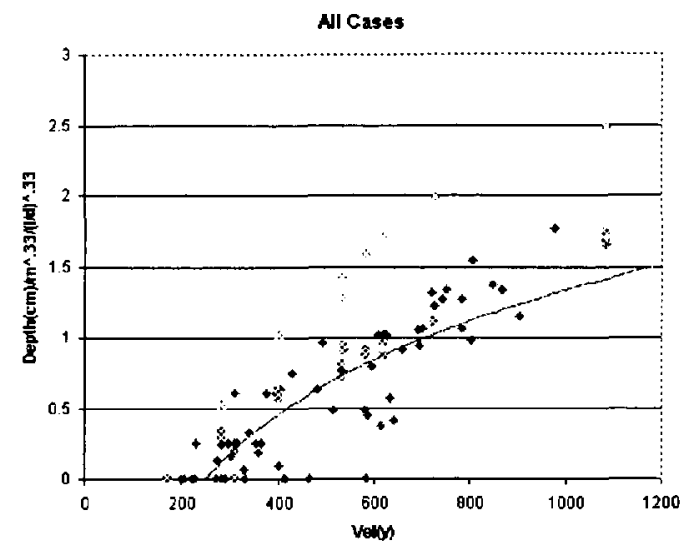

Figure 15. Same as Figure 14, but with fitting formula points shown as green o's.

\section{VALIDATION SUMMARY}

The smooth particle code SPHC was compared against experimental data from 1999 Southwest Research Institute foam-on-tile experiments. Ten shots were analyzed in detail, representing several different projectile sizes, velocities, and impact angles. In nine of the cases, the code results agreed closely with the experimental measurements of crater depth. In one remaining shot the results diverge, but the shot appears to be atypical when compared with other similar shots in the program. This exception exceeds the kind of variation to be expected in actual impacts on tiles with varying degrees of wear, and probably represents an experimental error of some kind.

Analysis of the test data shows the normal variation to be expected in actual flight tiles during their use cycles. This variation is about $+/-0.5 \mathrm{~cm}$ in the depth of the damage, or about $+/-10 \%$ of the tile thickness. Examination of the model results shows that the model probably reproduces the average damage to better accuracy than this inherent experimental scatter.

We conclude that the code results agree with the experimental data to within about $10 \%$.
Comparison of the SPHC results with those of the $\mathrm{R} \& \mathrm{C}$ fitting formula shows that the models fit the data much betier than that formula, and could be used to estimate damage to a greater degree of reliability. To facilitate this use of the model data, a new fitting formula has been derived for this set of experimental data. A more general formula could be obtained from a more extensive model series, and for various other TPS configurations to compare durability. This type of derivation would require hundreds of models to be run (each model takes about 1 hour, so this is certainly possible), and could be a subject for future work.

\section{APPLICATION TO STS-107 IMPACTS}

\section{Summary of Tile Test Simulations}

Table 3 summarizes the parameters and results for all of the shuttle tile impact tests simulated using SPHC. Cases 1-5 were pre-test configurations. This table numbers the cases by final shot sequence, plus a note of which edge of the projectile first struck the tile, e.g. " 3.5 " indicates that the 3.5-inch edge of the projectile made the initial impact. Tests " $5 b$ " were run with reduced density and increased block size as implemented for the actual test. The $5 \mathrm{~b}$ tests are therefore comparable to the actual experiments, and should indicate the effects of the lower

Table 3. Tile Impact Test Simulations

\begin{tabular}{|c|c|c|c|c|c|c|c|c|}
\hline \multirow[b]{2}{*}{ Case } & \multirow[b]{2}{*}{$\begin{array}{l}\text { Angle } \\
\text { deg }\end{array}$} & \multicolumn{3}{|c|}{ Foam } & \multicolumn{4}{|c|}{ Damage } \\
\hline & & $\begin{array}{l}\mathrm{Vel} \\
\mathrm{ft} / \mathrm{s}\end{array}$ & $\begin{array}{l}\text { Proj } \\
\text { Size }\end{array}$ & $\begin{array}{l}\text { Den } \\
\mathrm{lb} / \mathrm{ft}^{3}\end{array}$ & $\begin{array}{l}\text { Depth } \\
\text { in }\end{array}$ & $\begin{array}{l}\text { Vol } \\
\text { in }^{3}\end{array}$ & $\begin{array}{l}\text { W } \\
\text { in }\end{array}$ & \\
\hline $1-3.5$ & 5 & 700 & I & 2.4 & & 10 & 3.5 & \\
\hline $2-5.5$ & 5 & 700 & I & 2.4 & 2 & 10 & 5.5 & \\
\hline $3-11.5$ & 5 & 700 & II & 2.4 & 0.71 & 65 & 11.5 & 16 \\
\hline $4-5.5$ & 8 & 710 & III & 2.4 & 0.6 & 40 & 5.5 & 12 \\
\hline $4-11.5$ & 8 & 710 & III & 2.4 & 0.47 & 30 & 11.5 & 24 \\
\hline $5-5.5$ & 13 & 775 & III & 2.4 & 1.1 & 90 & 5.5 & 30 \\
\hline $5-11.5$ & 13 & 775 & III & 2.4 & 1.2 & 110 & 11.5 & 16 \\
\hline $5 b-5.5$ & 13 & 775 & IV & 2.0 & 0.78 & 55 & 5.5 & 25 \\
\hline $5 b-11.5$ & 13 & 775 & IV & 2.0 & 1 & 58 & 11.5 & 10 \\
\hline H-11.5 & 23 & 775 & III & 2.4 & 1.38 & 150 & 11.5 & 20 \\
\hline \multicolumn{2}{|c|}{$\begin{array}{l}\text { Projectile sizes: } \\
\text { (inches) }\end{array}$} & \multicolumn{6}{|c|}{$\begin{array}{l}\text { I }: 3.5 \times 11.5 \times 21.25 \\
\text { II }: 5.5 \times 11.5 \times 28.5 \\
\text { III }: 5.5 \times 11.5 \times 19 \\
\text { IV }: 5.5 \times 11.5 \times 23\end{array}$} & \\
\hline
\end{tabular}

density foam used in the experiments. Finally, test " $\mathrm{H}$ " assessed the effects of a larger impact angle on tile damage.

\section{Main Landing Gear Door Tests}

Five experiments were shot on the main landing gear door (MLGD) test panel in April-May, 
2003. All five exhibited very little damage to the tiles.

Prior to the shots, test parameters were distributed to our analysis team. Using these parameters, pre-test models were run with various codes, including SPHC. The parameters and results of these tests are summarized in Table 3, cases 1-5. At about the time of the fifth test, it became known that the foam density was actually $20 \%$ lower than that given in the specifications. To "allow for" this discrepancy, the foam projectiles being shot were made somewhat larger than specified. To see if the correction was working, the model for case 5 was rerun with only these changes (case 5b). This could be considered a "post-test" result, since other adjustments (such as slightly adjusting the velocity and angles), would have had very little effect.

The foam model used for these runs was a "softelastic" model for BX-250 foam. In this model the density was $2.4 \mathrm{lb} / \mathrm{ft}^{3}$, the elastic modulus 73.22 Mdynes $/ \mathrm{cm}^{2}$, the yield strength $5.0 \mathrm{M}$ dynes $/ \mathrm{cm}^{2}$, the tensile strength $5.2 \mathrm{Mdynes} / \mathrm{cm}^{2}$, and the maximum elongation at fracture $14.5 \%$. Poisson's ratio was set to the experimental value of 0.07 . The "crush" equation of state was used with an initial porosity of $92 \%$, and a surface friction coefficient of 0.10 . Static compression tests clearly indicate that the foam does not recover from compression cycles. The "elastic" behavior observed in the impact experiments is probably due to the effects of pore pressure.
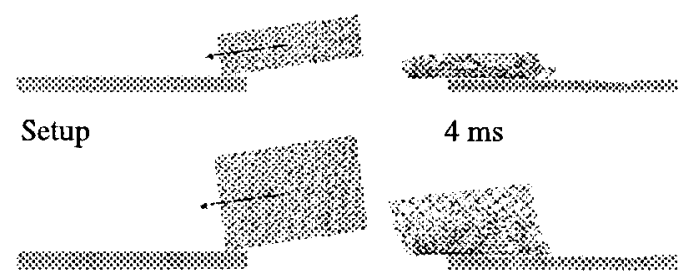

$4 \mathrm{~ms}$

Figure 16. Case 4 impact simulations.

The MLGD pre-test models all produced similar results. Figure 16 shows two "clocking angle" simulations for Case 4, run in 2D for maximum resolution. The top images show the case with the long edge impacting the tiles; the lower image shows the case for the short edge impacting. The impact angle for this case is 8 degrees, and the velocity $710 \mathrm{ft} / \mathrm{s}$. Five tiles were modeled, with the initial impact point in the center of the first tile.
In both cases the maximum damage occurred about 6 inches beyond the impact point, and this damagc was minimal. The validation models discussed previously indicate that in cases of this sort the experiments will probably indicate "no damage". This is because the full strength of the RCG glass coating cannot be modeled adequately by the single layer of particles used, so models with indentations of less than about 1 cm will most likely fail to crack the RCG layer, and show zero damage in the experiments. We observe a large amount of scatter in the test results near the damage threshold, probably caused by variations in the RCG strength, so a close agreement between models and experiments in this regime is not expected in any case.

Effect of Brittle Foam

To simulate the effects of pore pressure on the impact, several models were run with increased volumetric pressure terms, while keeping the deviatoric (strength) components unchanged. For these tests the pressure followed the usual crush curve, but was increased by a factor of three.

Figure 17 shows the result for case 3 with the increased pressure at $5 \mathrm{~ms}$. In this case several prominent cracks have developed in the foam structure. The main result of these cracks is to allow the rear portions of the foam block to impact the same tiles as the forward portions, rather than be deflected (bounced) out of the way, as with the elastic foam cases. In this figure the rear lower portion of the foam block is causing much more damage to the tiles than normally observed. About $2 / 3$ of the thickness of the LI-900 layer is removed, with damage extending to the SIP layer at points. This is probably a serious damage condition.

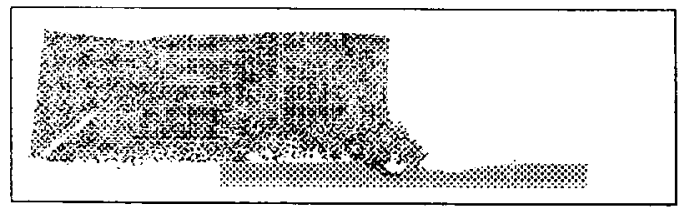

Figure 17. Case 3 with brittle foam, at $5 \mathrm{~ms}$, colored on region.

Any process that results in brittle foam, such as cryogenic conditions, can cause this effect. It was unexpected that increased pore pressure would cause brittle cracking, but these models show clearly that this is true. The mechanism is probably a decrease in the relative importance of 
the deviatoric terms, which contribute to the elasticity of the foam.

\section{Effect of Rotating Foam}

It is believed that the foam impactor on STS 107 was rotating at a rate of at least $17 \mathrm{rps}$. For this size object, 17 rps translates into transverse velocities of about $100 \mathrm{ft} / \mathrm{s}$ at the outer edges of the block. Although small compared to the translational velocity of $700-800 \mathrm{ft} / \mathrm{s}$, this is not small when compared to the normal component of this velocity, which is about $100 \mathrm{ft} / \mathrm{s}$ at a 5 degree impact angle. Rotation can thus approximately double the normal velocity, and perhaps also the transient normal force of impact at the instant of first contact.

To test the effect of a rotating block, Case 4 was rerun with forward and reverse rotation speeds of $17 \mathrm{rev} / \mathrm{s}$. The initial setup is shown in Figure 18.

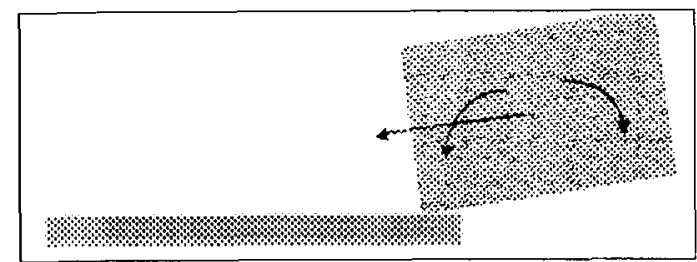

Figure 18. Case 4 setup showing the added rotation velocities.

In both cases the damage was less than in the non-rotating case. For the reverse, the forward edge impact velocity is reduced, and the impact is spread out over a larger area, so this result is expected. But what about the forward rotation case? In this case the normal velocity is nearly doubled, and the damage should be increased. What is happening here appears to be a reduction of the frictional interaction, resulting in a sort of "rolling" of the foam block, instead of a head-on impact, which, again, has the effect of spreading out the area of impact and reducing the depth of the resulting damage.

It is probable that for other impact parameters, or frictional interaction models, the rotational energy would increase the damage, but for these two cases the effect was just the opposite. This suggests that the rotational damage increase will be minimal for most cases.

We note in passing that SPHC has been designed to conserve angular momentum for all rotational cases, including large angle rotations as seen here. Many codes use a linear strength model (tensor model), which will not be able to model rotations of more than a few degrees accurately.

\section{Large Angle Case: 23-Degree Impact}

Since all of the experimental cases produced minimal damage (using elastic foam), a valid question is "How large an impact angle is needed to produce appreciable damage to the tile TPS system?". To address this question, the wide edge clocking angle of Case 4 was rerun with a 23 degree impact angle, and all other parameters kept constant. Figure 19 shows the initial setup for this test case and the results at $4 \mathrm{~ms}$.

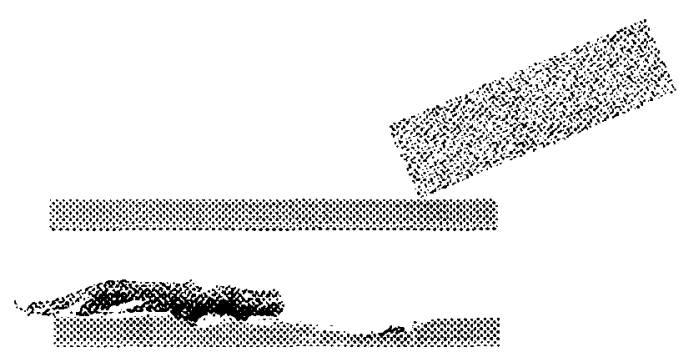

Figure 19. Large impact angle case.

Clearly, considerable damage has been done to the tile, and the foam is nearly completely damaged as well (in contrast to the shallow angle impacts, in which the foam block generally emerges almost intact). Damage to the tile extends into and through the SIP layer and would probably compromise the TPS system on reentry.

We conclude that impacts at angles of about 20 degrees or more for this size and velocity of foam block should be considered a threat to the integrity of the TPS system.

\section{Effect of Lower Foam Density}

To test the effect of the lower foam density actually shot in the experiments $\left(2.0 \mathrm{lb} / \mathrm{ft}^{3}\right.$, as opposed to the "nominal" density of $2.4 \mathrm{lb} / \mathrm{ft}^{3}$ ), Case 5 was rerun with the lower density and a larger foam particle, as in the experiment (Case $5 b)$. The results are summarized in the Table 3 above. For both cases the crater depth was lower in the low density case, and the crater volume was lower by about a factor of 2 for the low density/larger block scenario. It is thus probable that the low density foam used in the experiments resulted in less damage than expected for the nominal density. The actual density of the foam in STS 107 (as estimated by NASA) could have been as high a $3.0 \mathrm{lb} / \mathrm{ft}^{3}$. 


\section{CONCLUSIONS}

We take the following conclusions from this investigation:

1. Foam collisions with the tile TPS on the shuttle MLGD or Wing Acreage at very shallow angles (5-10 degrees) and velocities of $700-800 \mathrm{ft} / \mathrm{s}$ do very little damage to the tile TPS system.

2. Foam collisions at about 20 degrees or larger angles could severely damage the tiles.

3. Using a low density foam significantly reduced the damage potential in the experiments, and using a larger foam projectile did not offset this problem. Thus, experimental shots 1-5 probably underestimate damage for the STS-107 scenario.

4. Increasing the internal pressure of the foam (due, perhaps, to pore pressure), tends to cause brittle fracture in the foam, and increases the damage to the tiles.

5. Rapid rotation of the foam projectile could, in principle, increase the damage significantly, but in two test cases shown here the damage was actually less with rotating foam. This effect probably depends on the details of the collision, and the effects of friction at the foam/tile surface.

\section{REFERENCES}

[1] Stellingwerf, R. F., "The SPH_C Manual, User's Guide, Programmer's Guide, Technical Guide, Function Reference, Test Cases," Mission Research Corporation Report, AMRC-N-384.1$384.5,1989 \mathrm{a}$.

[2] Stellingwerf R. F., "Boundary Condition Tests Using Smooth Particle Hydrodynamics," Mission Research Corporation Report, MRC/ABQ-N-426, 1989b.

[3] Stellingwerf R. F., "Blast Wave Stability in Nuclear Explosions I," Mission Research Corporation Report, MRC/ABQ-R-1254, 1990a.

[4] Stellingwerf, R. F., "Smooth Particle Hydrodynamics," Advances in the FreeLagrange Method, Trease, Fritts, and Crowley, eds., Springer Verlag, p. 239, 1990 b.
[5] Stellingwerf R. F. and Peterkin, R. E., "Smooth Particle Magneto-hydrodynamics," Mission Research Corporation Report, MRC/ABQ-R-1248, 1990.

[6] Wingate, C. A. and Stellingwerf, R. F., "Los Alamos SPHINX Manual, Version 7.6," Los Alamos National Laboratory Report, LA-UR-932476, 1993.

[7] Mandell, D.A., Wingate, C. A., and Stellingwerf, R., F., "Prediction of Material Strength and Fracture of Brittle Materials Using the Sphinx Smooth Particle Hydrodynamics Code," Proceedings of the 10th ASCE Engineering Mechanics Conference, Boulder, CO, May 21-24, 1995.

[8] Stellingwerf, R. F., Robinson, J. H., Piekutowski, A.J., "Models and Experiments of Hypervelocity Impact of Orbital Debris on Thin Aluminum Targets," HVIS paper, in press, 2003.

[9] Stellingwerf, R. F., "Smooth Particle Hydrodynamics Code Analysis of STEPAIRSEDS Tether Material," NASA Project Report - H-34333D, 2001.

[10] Robinson, J. H., "Orbital Debris Impact Damage to Reusable Launch Vehicles," The International Journal of Impact Engineering 23, p. 783, Pergamon, 1999.

[11] Johnson, G. R., Stryk, R. A., Beissel, S. R., "SPH for High Velocity Impact Conditions," Computer Methods in Applied Mechanics and Engineering, pp. 347-373, 1996.

[12] Goodlin, D., L, "Orbiter Tile Testing," SwRI Project Number 18-7503-005, 1999.

[13] Forsberg, K., Center for System Management, preprint, 2001.

[14] Richardson, A. J., Chou J. C., Rockwell Report 280-106-85-005, 1985.

[15] Rand, J. L., Norton, D.J., NASA Final Report on contract NAS-9-15962, (no date). 